\title{
A MATHEMATICAL MODEL ON MALARIA INFECTION AND ITS SPREAD OVER A GIVEN POPULATION
}

\author{
C. E. ONWUKWE, J. A. UGBOH AND J. N. EZEORAH
}

(Received 7, November 2008; Revision Accepted 2, February 2009)

\begin{abstract}
This work focused on the modeling of malaria infection. Three kinds of models were considered; the first on the entire given population without control measure, the second include a control measure, and the third divides the population into adults and children with a control measure. The last model is considered the best model for the malaria infection in a growing population with control measure.
\end{abstract}

KEYWORDS: Modeling malaria infection, Control Measure

\section{INTRODUCTION}

We are developing mathematical models to better understand the transmission and spread of malaria. We modeled the disease using ordinary differential equations (ODEs) where humans and mosquitoes interact and infect each other. This model was used to determine the rate of spread of malaria when there is no control measure and when there is control measure. Malaria is an infectious disease caused by the Plasmodium parasite and transmitted between humans through the bite of the female Anopheles mosquito. An estimated $40 \%$ of the world's population lives in malaria endemic areas. It kills about

$0.7-2.7$ million people a year,75\% of whom are African children (WHO, 1986). The incidence of malaria has been growing recently due to increasing parasite drug-resistance and mosquito insecticide-resistance (WHO,1986). Therefore, it is important to understand the important parameters in the transmission of the disease and develop effective solution strategies for its prevention. Mathematical modeling of malaria began in 1911 with Ross and major extensions were described by MacDonald in 1957(Macdonald,1968; \& Halloran et al, 1989). By the 1970s, it was clear that Macdonald's model could be greatly improved by adding explicit considerations of human immunity, at which point, as part of the Garki project in Nigeria, Dietz and Molineaux developed a more sophisticated model. That model, in their words, did a "fairly realistic" job of simulating malaria epidemiology at Garki, given entomologic inputs, and provided conditional, comparative forecasts for several specific interventions (Molineaux \& Gramiccia ,1980). In the 1980s, Halloran and others took another step by explicitly considering the population- level effects of potential stage-specific vaccines (Halloran, Struchiner \& Spielman,1989). Now that malaria research and malaria control are beginning to gain attention again, we should focus more efforts and resources towards pragmatic, intervention focused modeling. We must make sure that malaria research and malaria control benefit more directly from the best tools available (McKenzie, 2000,McKenzie et al, 2002 and Samba, 2001). Recently, Ngwa and Shu (2000) proposed an ODE model for the spread of malaria.

\section{DEFINITION OF TERMS}

In this work, the following notations shall be used;

$Q(t)=$ Total population of people at time $t$

$\mathrm{H}(\mathrm{t})=$ Total number of susceptible at time $\mathrm{t}$

$\mathrm{H}_{1}(\mathrm{t})=$ Population of susceptible children

$\mathrm{H}_{2}(\mathrm{t})=$ Population of susceptible adults

$N(t)=$ Total number of infected at a time $t$ (infectious)

$A(t)=$ Total number of infected adults at a time $t$

$\mathrm{R}(\mathrm{t})=$ Total number of infected children at a time $\mathrm{t}$

$M=$ Mosquito biting rate that caused infection

$\mathrm{M}_{2}=$ Rate of mosquito bite in adults that caused infection.

$\mathrm{M}_{1}=$ Rate of mosquito bite in children that caused infection.

$B=$ blood transfusion rate that caused infection.

$M_{b}=$ rate of mingling of blood during birth that caused infection.

$\mathrm{P}=$ rate of exchange of material through placenta that caused infection.

$a=$ rate of cure of infection as a result of control.

$\mathrm{a}_{2}=$ rate of cure of infection in adult as result of control.

C. E. Onwukwe, Department of Maths/Stats \& Computer Science, University of Calabar, Calabar.

J. A. Ugboh, Department of Maths/Stats \& Computer Science, University of Calabar, Calabar.

J. N. Ezeorah, Department of Maths/Stats \& Computer Science, University of Calabar, Calabar. 
$a_{1}=$ rate of cure of infection in children as a result of control.

However, in this research work, we shall assume that $\mathrm{B}, \mathrm{M}_{\mathrm{b}}$, and $\mathrm{P}$ can easily be checked and controlled by medical practitioners. Thus, the only mode of spread of infection that will be treated here is mosquito.

\section{MODEL ASSUMPTION}

In this work, the following modeling assumptions are made;

i. There is at least an individual with the infection in the given population.

ii A susceptible becomes an infected immediately after transmission.

iii. The disease is spread by mosquito bite. .

iv. The effect of migration, births and death are negligible.

$v$. The disease is transmitted by close proximity between an infected and susceptible.

vi. The total population $Q(t)$ is divided into two groups; susceptible $H(t)$ and infected $N(t)$.

vii. $\quad$ The infection is cured at a rate 'a' proportional to the number of infectious.

viii. Children infective are cured at a rate $a_{1}$ proportional to their total number and adults infectious are cured at a rate $a_{2}$ proportional to their total number.

ix. New infectious are added to the adult population at a rate $a_{2}$ proportional to the total number of adult susceptible $\left(\mathrm{H}_{2}(\mathrm{t})\right)$ and children infectious are added to the children population at a rate $\mathrm{a}_{1}$ proportional to the total number of children susceptible $\left(\mathrm{H}_{1}(\mathrm{t})\right)$ and adult infectious.

MALARIAL MODEL 1: Mosquito bite as the only determining factor:

Consider a population of infectious N,M- the rate of mosquito bite that cause infection, then the change in the number of infectious is directly proportional to the number of mosquito bites and also directly proportional to the number already infected. From assumptions (i)-(v), we have that:

$\frac{d N}{d t} \propto M N \Rightarrow \frac{d N}{d t}=k M N$ where $\mathrm{k}$ is the constant of proportionality. Setting $\mathrm{k}=1$, we have

$\frac{d N}{d t}=M N$

Separating variables gives

$\frac{d N}{N}=M d t$.

Integrating both sides gives

$\int \frac{d N}{N}=\int M d t$

$\Rightarrow \ln N=M t+c$.

Taking exponential of both sides gives

$e^{\ln N}=e^{M t+c}$

$\Rightarrow N=e^{M t+c}$

$\Rightarrow N=e^{M t} \times e^{c}$.

Let $e^{c}=\mathrm{N}_{0}=$ initial number of infective then, at time $\mathrm{t}$;

$N(t)=N_{0} e^{M t}$

\section{INTERPRETATION OF MODEL}

From the model $N(t)=N_{0} e^{M t}$, if $\mathrm{N}_{0}$ is a constant and $\mathrm{M}>0$ then the number of infectious will grow exponentially over time as shown in fig 1 


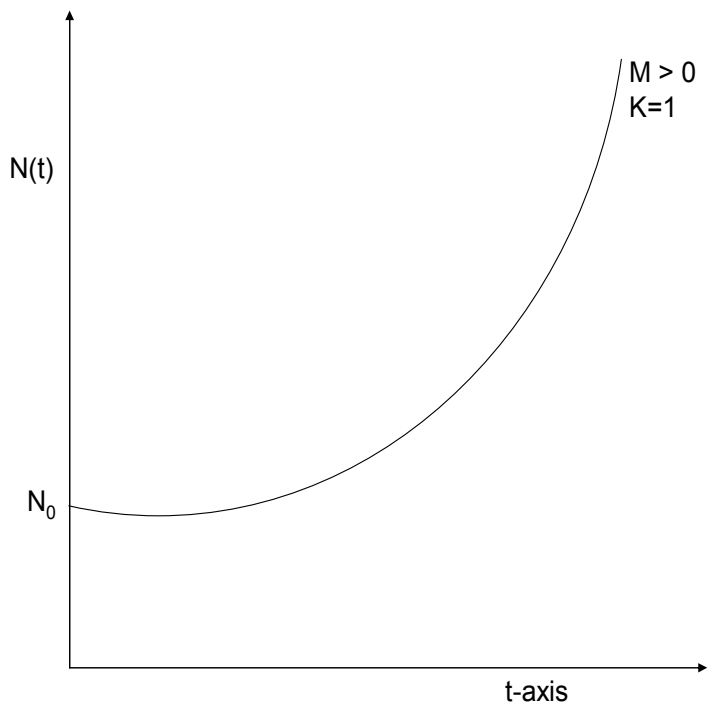

Fig. 1

If $M$ is equal to zero then the number of infective over time will be equal to the initial number $N_{0}$ i.e. $N(t)=N_{0}$

MALARIA MODEL2: Mosquito bite and cure as the determining factors.

Consider a population of infectious N. From assumption (i) - (v) and (vii), we have that the rate of infectious is directly proportional to number of infectious and the difference between the control and the biting rate; i.e

$\frac{d N}{d t} \propto N(M-a) \Rightarrow \frac{d N}{d t}=k N(M-a)$ setting $\mathrm{k}=1$ we have

$\frac{d N}{d t}=-a N+M N$

$\frac{d N}{d t}=N(M-a)$

The solution of (3) is obtained in a manner similar to that of (1), and is given by

$N(t)=N_{0} e^{(M-a) t}$

\section{INTERPRETATIONS}

From the model $N(t)=N_{0} e^{(M-a) t} \quad$ : if $\mathrm{M}>>$ a, the number of infectious will grow exponentially over time. The infection, in this case, will spread at a very fast rate over the entire population leaving nearly nobody untouched. This is illustrated in fig.2.

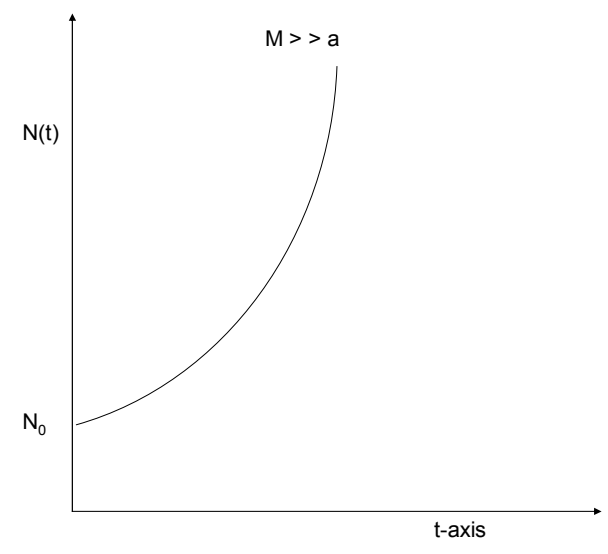

Fig.2 
If $M=a$, then, the number of infected at any time $t$ will be equal to the initial number i.e. $N(t)=N_{0}$. In this case, there is no spread. This is the case as in Model 1 when $M=0$. If $M<<a$, then the infection will diminish over time as illustrated in Fig. 3.

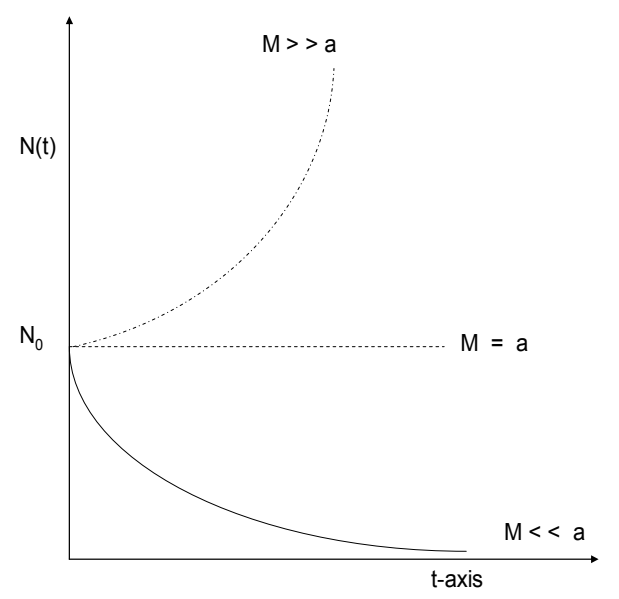

Fig.3

The three curves in Fig. 3 show the infection rates when $M>>a, M=a$, and $\mathrm{M}<<$ a.

MALARIA MODEL 3. Mosquito bite and cure as the determining factors.

Consider a population $Q$ from assumption (vi), (viii) and (ix), we have that;

$\frac{d A}{d t}=-a_{2} A+M_{2} H_{2}(t)$ or

$\frac{d A}{d t}+a_{2} A=M_{2} H_{2}(t)$

and

$\frac{d R}{d t}=-a_{1} R+M_{1} H_{1}(t)$ or

$\frac{d R}{d t}+a_{1} R=M_{1} H_{1}(t)$

Solving equation 5 by the method of integrating factor, (Kreyszig, 2004)

we let $\mu_{2}=e^{\int a_{2} d t}=e^{a_{2} t}$ to be the integrating factor, and obtain

$$
A(t)=G_{2}(t)+C e^{-a_{2} t}
$$

$$
\text { where } G_{2}(t)=\frac{\int e^{a_{2} t} H_{2}(t) d t}{e^{a_{2} t}} \text { and } C \text { a constant of integration }
$$

Similarly, equation (6) yields

$$
R(t)=G_{1}(t)+D e^{-a_{1} t}
$$

$$
\text { where } G_{1}(t)=\frac{\int e^{a_{1} t} H_{1}(t) d t}{e^{a_{1} t}} \text { andD, a con } \$ \text { ant }
$$

Since $A(t)+R(t)=N(t)$ we have

$N(t)=G_{1}(t)+G_{2}(t)+D e^{-a_{1} t}+C e^{-a_{2} t}$

As $t$ tends to infinity (or for large $a_{1}$ and $a_{2}$ ) the last two terms drops out and we have 


$$
N(t)=G_{1}(t)+G_{2}(t)
$$

where $G_{1}(t)$ is a contribution from children and $G_{2}(t)$ is from Adult

From model 2, the form of $\mathrm{H}_{1}(\mathrm{t})$ and $\mathrm{H}_{2}(\mathrm{t})$ are respectively

$$
\begin{aligned}
& H_{1}(t)=h_{1} e^{\left(a_{1}-m_{1}\right) t} \\
& H_{2}(t)=h_{2} e^{\left(a_{2}-m_{2}\right) t}
\end{aligned}
$$

where $h_{1}$ and $h_{2}$ are respectively the initial children and adult susceptible.

\section{DISCUSSION}

The malaria model $N(t)=N_{0} e^{M t}$, predicts a very rapid spread of the disease. This is so because there is no control measure adopted. Thus, within a short time, virtually every body in that population will be infected. Thus, a high mortality rate will be experienced in that population. This situation is redressed with the help of the quantity ' $a$ ' in model $N(t)=N_{0} e^{(M-a) t}, \mathrm{a}_{2}$ in $A(t)=G_{2}(t)+C e^{-a_{2} t}$ and $\mathrm{a}_{1}$ in $R(t)=G_{1}(t)+D e^{-a_{1} t}$

It predicts that a cure of the disease will help reduce the rate of spread of the malaria parasite. In particular, if $a>>m$, then equation (4) predicts that the number of infected over time will drastically reduce.

In equations (7) and (8) as $t$ tends to infinity (or for large $a_{1}$ and $a_{2}$ ) the last two terms drop out and we have $N(t)=G_{1}(t)+G_{2}(t)$ where $G_{1}(t)$ is a contribution from children and $G_{2}(t)$ is from Adult. Also from the $H_{1}(t)=h_{1} e^{\left(a_{1}-m_{1}\right) t}$ we deduce that as treatment rate $\mathrm{a}_{1}$ outweighs the bite rate $\mathrm{m}_{1}$ the children susceptible population increases and the reverse is the case when $m_{1}$ is far greater than $a_{1}$. The same goes for adult susceptible.

\section{CONCLUSION}

Having seen the above analysis, it is obvious that cure is one of the major ways of fighting the spread of the malaria parasite. Therefore, it will be wise if infected individuals are treated and on time. This will help kill the parasite in the body so that when the mosquito bites, it will not see parasite to suck and transmit to another person. However, other control measures as mentioned earlier will still be very useful.

\section{REFRENCES}

Halloran, M. E, Struchiner, C. J, Spielman, A., 1989. Modeling malaria vaccines. II: Population effects of stage-specific malaria vaccines dependent on natural boosting. Math Biosci 94: 115-149.

Knell, A. F., 1997. Malaria: A publication of the Tropical Programme of the Welcome Trust. Oxford; Oxford University Press.

Kreyszig, E., 2004. Advanced Engineering Mathematics; $8^{\text {th }}$ edition. John Wiley and Sons Inc. New York

Macdonald G., 1957. The Epidemiology and Control of Malaria. Oxford, United Kingdom: Oxford University Press.

Macdonald, G., 1968. The dynamics of malaria. Bull World Health Organ 38: pp743-755. Geneva.

McKenzie F.E., 2000. Why model malaria? Parasitol Today 16:511-516.

McKenzie F. E, Baird J. K, Beier J. C, Lal A. A, Bossert W. H, 2002. A biologic basis for integrated malaria control. Am J Trop Med Hyg 67: 571-577.

Molineaux L, Gramiccia G., 1980. The Garki Project. Geneva: World Health Organization.

Ngwa G. A. and Shu W. S., 2000. Math. Comput. Modelling, 32:747-763.

Samba, E. M, 2001. The malaria burden and Africa. Am J Trop Med Hyg 64(suppl):

WHO, 1986. Malaria : WHO Expert Committee on Malaria, $18^{\text {th }}$ Report- Geneva,. 